\title{
E-values for effect heterogeneity and approximations for causal interaction
}

\author{
Maya B. Mathur*1, Louisa H. Smith², Kazuki Yoshida ${ }^{3}$, \\ Peng Ding ${ }^{4}$, and Tyler J. VanderWeele ${ }^{5}$
}

${ }^{1}$ Quantitative Sciences Unit and Department of Pediatrics, Stanford University, Palo Alto, CA

${ }^{2}$ Roux Institute, Northeastern University, Portland, ME

${ }^{3}$ Department of Medicine, Brigham and Women's Hospital and Harvard Medical School, Boston, MA

${ }^{4}$ Department of Statistics, University of California at Berkeley, Berkeley, CA

${ }^{5}$ Department of Epidemiology, Harvard University, Boston, MA

- Citation: Mathur MB, Smith LH, Yoshida K, Ding P, VanderWeele TJ (in press). Evalues for effect heterogeneity and approximations for causal interaction. International Journal of Epidemiology. Preprint retrieved from https://osf.io/h6pru/.

- Word count: 3,869

- Type of manuscript: Original Article

- Conflicts of interest: The authors declare that they have no conflicts of interest.

${ }^{*}$ Correspondence to: Maya B. Mathur (mmathur@stanford.edu), Quantitative Sciences Unit, 1701 Page Mill Road, Palo Alto, CA, 94304. 


\begin{abstract}
(204/250)
Background: Estimates of effect heterogeneity (i.e., the extent to which the causal effect of one exposure varies across strata of a second exposure) can be biased if the exposure-outcome relationship is subject to uncontrolled confounding whose severity differs across strata of the second exposure.

Development: We propose methods, analogous to the E-value for total effects, that help assess the sensitivity of effect heterogeneity estimates to possible uncontrolled confounding. These E-value analogs characterize the severity of uncontrolled confounding strengths that would be required, hypothetically, to "explain away" an estimate of multiplicative or additive effect heterogeneity in the sense that appropriately controlling for those confounder(s) would have shifted the effect heterogeneity estimate to the null, or alternatively would have shifted its confidence interval to include the null. One can also consider shifting the estimate or confidence interval to an arbitrary non-null value. All of these E-values can be obtained using the R package EValue.
\end{abstract}

Application: We illustrate applying the proposed E-value analogs to studies on: (1) effect heterogeneity by sex of the effect of educational attainment on dementia incidence and (2) effect heterogeneity by age on the effect of obesity on all-cause mortality.

Conclusions: Reporting these proposed E-values could help characterize the robustness of effect heterogeneity estimates to potential uncontrolled confounding.

Keywords: sensitivity analysis, bias analysis, confounding, interaction, effect modification, effect heterogeneity

\title{
KEY MESSAGES
}

- Effect heterogeneity estimates can be biased if the exposure-outcome relationship is subject to uncontrolled confounding whose severity differs across strata of the second exposure.

- We propose sensitivity analyses, analogous to the E-value, that characterize the severity of uncontrolled confounding strengths that would be required to "explain away" an estimate of effect heterogeneity.

- We provide an $\mathrm{R}$ package, EValue, to conduct all proposed sensitivity analyses. 


\section{BACKGROUND}

Estimates of effect heterogeneity (i.e., the extent to which the causal effect of an exposure, $X$, varies across strata of another variable, $Z$ ) can be biased if the exposure-outcome relationship is subject to uncontrolled confounding whose severity differs across strata of $Z(1,2)$. For example, suppose that in the $Z=1$ stratum, the exposure-outcome relationship is biased upward due to uncontrolled confounding, but that in the $Z=0$ stratum, the relationship is not biased. The uncontrolled confounding in the $Z=1$ stratum could, for example, spuriously increase the magnitude of the observed ratio or difference between the 2 strata's estimated exposure-outcome effects, thus increasing the observed estimate of effect heterogeneity. Such bias in effect heterogeneity estimates can be consequential in practice. For example, effect heterogeneity estimates on the additive scale can inform decisions of which individuals (i.e., which stratum of $Z$ ) to treat in order to most reduce disease caseload if treatment resources are limited (1). In this context, a biased estimate of effect heterogeneity could potentially suggest a treatment allocation scheme that fails to minimize, or even increases, overall caseload.

Although sensitivity analyses exist for causal interactions (i.e., effects of jointly manipulating $X$ and $Z$ versus each independently) (3), we are not aware of comparable methods for effect heterogeneity. We propose sensitivity analyses that characterize the severity of uncontrolled confounding strengths that would be required, hypothetically, to "explain away" an estimate of multiplicative or additive effect heterogeneity in the sense that controlling for those confounder(s) would have shifted the effect heterogeneity estimate to the null, or alternatively to would have shifted its confidence interval to include the null. We recommend reporting E-values for both the point estimate and the confidence interval; the latter is especially important for effect heterogeneity estimates, for which statistical precision is often considerably lower than for total effects. We also discuss "non-null" E-values required to shift the estimate or its confidence interval to any arbitrary value. These metrics are straight- 
forward extensions of the standard E-value for total effects, which represents the minimum strength of association, on the risk ratio $(R R)$ scale, that uncontrolled confounder(s) would need to have with the exposure, the outcome, or both, conditional on any measured and controlled confounders, to explain away the total effect $(4,5)$. As we describe below, it is equivalent to interpret the E-value as the minimum strengths of association that uncontrolled confounder(s) would need to have with both the exposure and the outcome, if these 2 strengths of association are taken to be of equal magnitude.

Like the standard E-value, our proposed E-values for effect heterogeneity do not require assumptions on the nature of the uncontrolled confounder(s) (4-6). That is, these metrics represent bounds under hypothetical worst-case confounding: they consider the maximum bias that could be generated by a given strength of confounder strengths, but actual uncontrolled confounders might not generate that much bias $(4,5)$. We also give alternative E-values that can be applied under the assumption that uncontrolled confounding operates in the same direction in each stratum of $Z$ ("unidirectional confounding"). We provide software to calculate all of these E-values, discussed below.

Details and reporting guidelines for the standard E-value are discussed elsewhere (4-7). The E-value has limitations, which have been discussed and debated elsewhere (8-11). For example, to avoid making assumptions about the prevalence or distribution of uncontrolled confounder(s), the E-value does not make use of potential known information about these and hence may understate the amount of confounding required to explain away an effect. Additionally, the E-value does not account for biases or threats to inferential validity other than uncontrolled confounding, such as measurement error, selective reporting, or uncontrolled multiple testing; we provide methods and recommendations regarding these issues elsewhere $(6,12-14)$. The same considerations and limitations apply for the analogs we propose. 


\section{SetTing And NOTATiOn}

All proofs, with formalized assumptions and definitions, appear in the Supplement. Let $Z \in\{0,1\}$ define strata between which the causal effect of $X \in\{0,1\}$ might vary, and let $Y_{x} \in\{0,1\}$ be a potential outcome when intervening to set $X=x$. (If $Z$ is categorical rather than binary, the same results can be applied to contrasts between 2 specified levels of $Z$.) We assume that the confounded estimate of effect heterogeneity is greater than the null (e.g., greater than 1 for multiplicative measures or greater than 0 for additive measures); otherwise, one can simply reverse the coding of $Z$ before applying the results below. We also assume that, other than the omission of uncontrolled confounder(s), the estimator used to obtain the confounded estimate of effect heterogeneity is correctly specified; that is, the estimate would have been unbiased for the causal estimand if the uncontrolled confounder(s) had in fact been controlled. For example, if the statistical interaction coefficient for $X \times Z$ in a regression model is taken to be the effect heterogeneity estimate, this requires assuming that $X$ does not affect $Z$, even if there were no uncontrolled confounding.

We define 2 sensitivity parameters for each stratum, called the "within-stratum confounding strengths". These are the same parameters used for the standard E-value, applied separately to each stratum of $Z$ (5). Within stratum $Z=z$, let $U_{z}$ denote uncontrolled confounder(s), defined as a set of one or more variables that would suffice to control for confounding of the exposure-outcome relationship in this stratum. As for the standard E-value, the same sensitivity parameters and results accommodate the possibility that $U_{z}$ includes one or more confounders of any type (e.g., binary, categorical, or continuous) and distribution. For example, if $U_{z}$ is binary, $R R_{U Y} \mid Z=z, X=x$ is simply the $R R$ relating $U_{z}$ to $Y$ within stratum $Z=z$. Precise interpretations of the sensitivity parameters when $U_{z}$ contains multiple confounders or is continuous are given elsewhere (6).

Within stratum $Z=z$ and for each stratum $x$ of $X$, define an $R R$ of $U_{z}$ on $Y$, maximized 
across all possible contrasts of $U_{z}$ :

$$
R R_{U Y \mid Z=z, X=x}=\frac{\max _{u} E\left[Y \mid Z=z, X=x, U_{z}=u\right]}{\min _{u} E\left[Y \mid Z=z, X=x, U_{z}=u\right]}, x \in\{0,1\}
$$

The sensitivity parameters $R R_{U Y \mid Z=1}$ and $R R_{U Y \mid Z=0}$ are then:

$$
R R_{U Y \mid Z=z}=\max \left\{R R_{U Y \mid Z=z, X=0}, R R_{U Y \mid Z=z, X=1}\right\}, z \in\{0,1\}
$$

Any confounders that are measured and controlled could also be conditioned throughout. These sensitivity parameters represent, within stratum $Z=z$ and considering both strata of $X$, the largest of the maximal $R R \mathrm{~s}$ of that stratum's uncontrolled confounders, $U_{z}$, on $Y$ conditional on $X$. The sensitivity parameters $R R_{X U \mid Z=1}$ and $R R_{X U \mid Z=0}$ are defined as:

$$
R R_{X U \mid Z=z}=\max _{u}\left\{\frac{P\left(U_{z}=u \mid Z=z, X=1\right)}{P\left(U_{z}=u \mid Z=z, X=0\right)}\right\}, z \in\{0,1\}
$$

Each parameter $R R_{X U \mid Z=z}$ represents, within stratum $Z=z$, the maximal $R R$ of $U_{z}=u$ for $X=1$ versus $X=0$ across strata of $U_{z}$.

We define the E-value for an effect heterogeneity point estimate as the minimum magnitude that at least one of the 4 within-stratum confounding strengths must have, on the $R R$ scale, such that fully controlling for confounding would have shifted the estimate to the null. Like the standard E-value, this E-value essentially sets the 4 confounding strengths equal to one another to obtain the required joint minimum for all of them. For the special case in which confounding is assumed to be unidirectional, we define E-values as the minimum value that at least one of the 2 confounding strengths in at least one stratum must have in order to explain away the effect heterogeneity.

To dispel a common misconception, we note that mathematically setting the confounding strengths equal to one another in this manner does not require assuming that, in reality, the confounding strengths actually are equal. The E-value is derived by considering all possible 
combinations of confounding strengths that could produce enough bias to explain away the effect heterogeneity, and then solving for the combination that minimizes the maximum of these confounding strengths (Supplement, Section 2.1). This unique combination, it turns out, is the one in which the confounding strengths are equal. If, in reality, there are uncontrolled confounders whose confounding strengths are not equal, the E-value still applies; it states that at least one of the confounding strengths must exceed the E-value in order to explain away the effect. Again, this interpretation is mathematically equivalent to considering confounding strengths of equal magnitude. Additionally, we note that setting the confounding strengths equal to one another does not require assuming that these associations arise from the same confounders in the 2 strata.

\section{Multiplicative EFFECT Heterogeneity}

We consider the causal estimand:

$$
R R_{E H}^{t}=R R_{X Y \mid Z=1}^{t} / R R_{X Y \mid Z=0}^{t}
$$

where $R R_{X Y \mid Z=z}^{t}=E\left[Y_{1} \mid Z=z\right] / E\left[Y_{0} \mid Z=z\right]$. Its confounded counterpart is:

$$
R R_{E H}^{c}=R R_{X Y \mid Z=1}^{c} / R R_{X Y \mid Z=0}^{c}
$$

where $R R_{X Y \mid Z=z}^{c}=E[Y \mid Z=z, X=1] / E[Y \mid Z=z, X=0]$. Again, any confounders that are measured and controlled can also be conditioned. (If multiplicative effect heterogeneity is measured on a different scale, such as the odds ratio or hazard ratio scale, then the estimate could be approximately converted to an $R R$ before applying the results below, as for the standard E-value (4).) Without making assumptions on the direction of confounding bias, 
the E-value required to shift $R R_{E H}^{c}$ to the null is:

$$
\text { E-value }=\sqrt{R R_{E H}^{c}}+\sqrt{R R_{E H}^{c}-\sqrt{R R_{E H}^{c}}}
$$

This E-value represents the minimum magnitude that at least one of the 4 within-stratum confounding strengths must have in order to explain away the effect heterogeneity (i.e., to have $R R_{E H}^{t}=1$ ). Equivalently, this E-value represents the minimum magnitude of all 4 within-stratum confounding strengths that would be required to explain away the effect heterogeneity, if all 4 confounding strengths are taken to be of equal magnitude. We use "magnitude" to indicate that the confounding strengths on the $R R$ scale are taken to be $\geq 1$ regardless of the direction of association, as for the standard E-value (4). In fact, this bound is attained when the effects within each stratum have bias of the same magnitude, but in opposite directions. It might be quite unlikely in practice that any given uncontrolled confounder has all 4 confounding strengths of equal magnitude, or that confounding bias operates in different directions in each stratum. We return to these points in Section 6.2. In the Supplement (Section 2.3), we establish connections between this bound and classical bounds on bias in total effects due to uncontrolled confounding $(15,16)$.

The E-value required to shift $R R_{E H}^{c}$ to a non-null value, $R R_{E H}^{t}$, rather than to the null can be obtained by replacing $R R_{E H}^{c}$ in Eq. (1) with $R R_{E H}^{c} / R R_{E H}^{t}$. The E-value required to shift the confidence interval to include the null or another specified value can be obtained by replacing $R R_{E H}^{c}$ above with the lower confidence interval limit. All of these E-values can be obtained using existing software that calculates E-values for total effects $(17,18)$ by simply performing the calculation using $\sqrt{R R_{E H}^{c}}$ rather than $R R_{E H}^{c}$ itself, and likewise for the confidence interval limit or $R R_{E H}^{c} / R R_{E H}^{t}$, because doing so is equivalent to applying Eq. (1).

If we instead assume unidirectional confounding, the E-value required to shift $R R_{E H}^{c}$ to 
the null is:

$$
\text { E-value }=R R_{E H}^{c}+\sqrt{R R_{E H}^{c}\left(R R_{E H}^{c}-1\right)}
$$

This represents the minimum magnitude that at least of one of the within-stratum confounding strengths must have in at least one stratum of $Z$ in order to explain away the effect heterogeneity. Equivalently, this E-value represents the minimum magnitude that both within-stratum confounding strengths must have in at least one stratum of $Z$, if both confounding strengths in that stratum are taken to be equal. This bound is attained when the effect within the other stratum is unbiased, and the expression is in fact equivalent to calculating the standard E-value for $R R_{E H}^{c}$.

\section{AdDitive EFFECT Heterogeneity}

Define the causal risk difference in stratum $Z=z$ and its confounded counterpart as:

$$
\begin{aligned}
& R D_{X Y \mid Z=z}^{t}=E\left[Y_{1} \mid Z=z\right]-E\left[Y_{0} \mid Z=z\right] \\
& R D_{X Y \mid Z=z}^{c}=E[Y \mid Z=z, X=1]-E[Y \mid Z=z, X=0]
\end{aligned}
$$

We consider the additive interaction contrast, $I C^{t}=R D_{X Y \mid Z=1}^{t}-R D_{X Y \mid Z=0}^{t}$, and its confounded counterpart $I C^{c}=R D_{X Y \mid Z=1}^{c}-R D_{X Y \mid Z=0}^{c}$ (19). Let $f_{z}=E[X \mid Z=z]$ be the prevalence of $X$ in stratum $Z$ and let $p_{z x}=E[Y \mid Z=z, X=x]$ be the outcome probability within the joint stratum $(Z=z, X=x)$. Then, without assumptions on the direction of confounding bias, the E-value required to shift $I C^{c}$ to the null is:

$$
\text { E-value }=g\left(\frac{1}{2\left(f_{1} p_{10}+f_{0} p_{01}\right)}\left\{\sqrt{\gamma^{2}+4\left(p_{10} f_{1}+p_{01} f_{0}\right)\left(p_{11}\left[1-f_{1}\right]+p_{00}\left[1-f_{0}\right]\right)}-\gamma\right\}\right)
$$


where $g(w)=w+\sqrt{w(w-1)}$ and $\gamma=p_{10}\left(1-f_{1}\right)-p_{11} f_{1}+p_{01}\left(1-f_{0}\right)-p_{00} f_{0}$. This bound is attained when the within-stratum effects are biased in opposite directions, but with potentially different amounts of absolute bias $\left(\left|R D_{X Y \mid Z=z}^{c}-R D_{X Y \mid Z=z}^{t}\right|\right)$ in each stratum. (This asymmetry arises because, in the additive case, the amount of absolute bias produced by a given fixed set of sensitivity parameters depends on nuisance parameters, such as exposure prevalences and outcome probabilities, that can differ between strata of $Z$. In contrast, for the multiplicative case, the amount of multiplicative bias is independent of any such nuisance parameters.) The Supplement provides a counterpart for the confidence interval, a generalization for shifting $I C^{c}$ to a non-null value, and E-values that apply if the direction of confounding bias is assumed to be unidirectional and positive, unidirectional and negative, or unidirectional with the direction unknown. All of these E-values for interaction contrasts can be obtained in R via EValue: : evalues. IC (17).

\section{APPliEd EXAMPles}

\subsection{Education and dementia}

Letenneur et al. (20) investigated the effect of low versus high education ( $\leq 7$ years of schooling versus $\geq 12$ years) on dementia incidence, additionally estimating effect heterogeneity by sex. They pooled data from population-based longitudinal studies of aged women $(n=3352)$ and men $(n=2395)$. In analyses that adjusted for baseline confounders (age, smoking, myocardial infarction, stroke, and study center), the authors estimated a strong association between low education and dementia incidence in women $(R R=3.78$ [95\% CI: 1.64, 8.72]), but an apparently much weaker association in men $(R R=1.09[0.61,1.94])$. The authors suggested that uncontrolled confounding might have produced this apparent effect heterogeneity. For example, they speculated that socioeconomic status, an uncontrolled confounder, might produce different biases for each sex.

On the multiplicative scale, we estimated $R R_{E H}^{c}=3.47[1.26,9.57] ; p=0.02$. Without 
making assumptions on the direction of uncontrolled confounding bias for each sex, the E-values for this estimate and its lower confidence interval limit were thus 3.13 and 1.49, respectively (from Eq. (1)). Thus, at least one of the 4 confounding strengths would need to be at least 3.13 on the $R R$ scale in order to explain away the effect heterogeneity, and would need to be at least 1.49 to shift the confidence interval to include the null. If we assume that uncontrolled confounding operated in the same direction for each sex, then the E-values for the point estimate and its lower confidence interval limit increase to 6.39 and 1.82, respectively (from Eq. (2)). Given these studies' control of several known confounders, it may be somewhat implausible that uncontrolled confounders, such as socioeconomic status, were strong enough to attain these confounding strengths, although such judgments would need to be informed by domain expertise.

On the additive scale, we estimated risk differences of $0.04[0.02,0.05]$ for women and $0.01[-0.01,0.02]$ for men, such that $I C^{c}=0.03[0.01,0.05] ; p=0.01$. This analysis does not adjust for confounders because Letenneur et al. (20) reported only unadjusted prevalences. Without assumptions on the direction of confounding bias, the E-values for the interaction contrast and its lower confidence interval limit were thus 2.54 and 1.44, respectively (from Eq. (3)). If we assume that uncontrolled confounding operated in the same unspecified direction for both sexes, then these E-values respectively become 3.30 and 1.63 (Supplement, Section $3.2)$.

\subsection{Obesity and all-cause mortality}

Winter et al. (21) meta-analyzed 8 longitudinal studies to investigate the extent to which effects of body mass index (BMI) on mortality differed by age ( $<65$ years versus $\geq 65$ years). The authors estimated that being obese $(\mathrm{BMI} \geq 30)$ versus normal weight $(20 \leq \mathrm{BMI}<25)$ was associated with increased mortality among participants aged $<65$ years $(H R=1.42$ [95\% CI: $1.22,1.65])$, but not among participants aged $\geq 65$ years $(H R=1.04$ [95\% CI: 0.91, 1.19]). Confounding control in the 8 studies was quite limited: for example, 5 studies did 
not control for comorbid health conditions, 2 studies did not control for smoking, 5 did not control for physical activity, and none controlled for diet or caloric intake.

On the multiplicative scale, we estimated $H R_{E H}^{c}=1.37[1.12,1.67] ; p=0.002$. Evalues can be applied to meta-analysis point estimates, in which case they represent average confounding strengths across studies $(13,22)$. Without making assumptions on the direction of uncontrolled confounding bias for each age group, the E-values for this estimate and its lower confidence interval limit were thus 1.61 and 1.30, respectively. If we assume that uncontrolled confounding operated in the same direction for each age group, then the Evalues for the point estimate and its lower confidence interval limit increase to 2.07 and 1.48, respectively. Given these studies' limited control of confounders whose associations with both obesity and mortality may be quite strong, it may be plausible that uncontrolled confounders could have the strengths of association indicated by the E-values. Furthermore, it may be plausible that the strength of confounding bias could differ by age group. For example, older individuals might be less physically resilient to comorbid conditions than younger individuals, such that having comorbid conditions might be more strongly associated with BMI or with mortality among older individuals. We could not conduct analyses on the additive scale given the statistics reported in the meta-analysis (21).

\section{Practical interpretation and Reporting of E-VAlues}

Elsewhere, we have provided recommendations on reporting E-values for total effects $(4,7)$ and provided caveats about potential misinterpretations $(11,23,24)$. These considerations apply to the present analogs as well; we comment here on only a subset of these considerations that are particularly pertinent to the setting of effect heterogeneity. 
6.1. Assessing the plausibility that confounding strengths attain the E-value As we have emphasized in the context of total effects, E-values must be interpreted in light of the quality of a study's existing control for confounding: it will be more plausible that there exists uncontrolled confounding as strong as is indicated by the E-value in a poorly-controlled study than a well-controlled study $(4,7)$. As a limitation of sensitivity analyses in general, it can be challenging to assess plausible strengths of association of uncontrolled confounder(s). This may be particularly so in the context of effect heterogeneity, for which the relevant confounding strengths are defined within strata of $Z$ rather than marginally. In the context of total effects, we have suggested listing specific variables that are thought to be uncontrolled confounders and, to help benchmark intuitions, reporting measured confounders' strengths of association with $X$ and with $Y$. Because E-values consider joint associations produced by potentially multiple uncontrolled confounder(s), above and beyond controlled confounder(s), it may be particularly informative to report confounding strengths for each measured confounder as well as for all measured confounders jointly (6). However, these empirical benchmarks must be interpreted carefully to avoid common misconceptions $(7,24)$. When applying E-values for effect heterogeneity, one could similarly report measured confounding strengths within strata of $Z$; for example, one could report the associations of each measured confounder, and of all measured confounders jointly, with $X$ and $Y$ for each stratum of $Z$. We acknowledge, though, that comparing the E-value to such benchmarks may be difficult because confounders, whether measured or not, can differ substantially in their associations with the exposure and outcome as well as in the strengths of these associations in each stratum. When additional information about uncontrolled confounders is available, the E-value could be supplemented or replaced by more precise sensitivity analyses; we return to this point in Section 6.2. 


\subsection{Interpreting E-values in light of their mathematical conservatism}

As noted above, like the standard E-value for total effects (4), our proposed analogs avoid making assumptions about the prevalence or distribution of uncontrolled confounder(s) by not incorporating any sensitivity parameters regarding these properties. Therefore, E-values might understate the amount of confounding required to explain away the effect or effect heterogeneity. For example, if $U_{z}$ is a binary variable, then given its confounding strengths, the bias it produces in stratum $Z=z$ would be maximized if $E[U \mid Z=z, X=1]=1$ and $E[U \mid Z=z, X=0]=0$ (or vice versa). The E-value is conservative in that it allows for this extreme, and sometimes implausible, possibility.

In some settings, one might wish to conduct a sensitivity analysis for specific uncontrolled confounder(s) whose prevalence conditional on $X$ or conditional on $Z$ is known. One could then obtain a more precise sensitivity analysis by applying methods that do incorporate information about prevalences. For effect heterogeneity, one could apply existing sensitivity analyses for uncontrolled confounding that incorporate prevalences or other external information (reviewed in (25)) to bound the bias in each stratum of $Z$ separately. Analogous bounds incorporating prevalences exist for causal interaction (3). Of course, the disadvantage of incorporating prevalences is that, if the specified prevalences are incorrect or if there exist other uncontrolled confounder(s) with prevalences other than those specified, bounds obtained by incorporating prevalences may give a false impression of robustness to uncontrolled confounding. Additionally, methods that involve specifying numerous sensitivity parameters could introduce additional "researcher degrees of freedom", such that researchers could potentially search for combinations of parameters that produce attractive results of sensitivity analyses (4). For these reasons, in the context of total effects, we suggested that when external information is available regarding uncontrolled confounder(s), one might first report the E-value, because despite its conservatism, the E-value might nevertheless be large enough to suggest robustness to uncontrolled confounding, even without making use of external 
information (25). One could then supplement this mathematically conservative analysis with additional sensitivity analyses that do incorporate external information (25). The same considerations also apply when considering effect heterogeneity.

A second form of conservatism arises specifically in the context of E-values for effect heterogeneity. Namely, as noted above, the general bound in Eq. (1) is attained when the effects in each stratum of $Z$ have bias of the same magnitude, but in opposite directions. In some scientific contexts, it might not be plausible that uncontrolled confounder(s) could in fact produce bias in opposite directions, and in these settings, the general bound in Eq. (1) might again understate the amount of confounding required to explain away the effect heterogeneity. However, in other contexts, it might be quite plausible that confounding bias could operate in different directions, because either the uncontrolled confounder(s) themselves, or alternatively the direction of their effects on $X$ or on $Y$, could differ between strata. Consider a hypothetical study examining effect heterogeneity between men and women $(Z)$ in the effects of smoking $(X)$ on all-cause mortality $(Y)$. Suppose the study excluded heavy drinkers but did not control for moderate versus low alcohol consumption $\left(U_{z}\right)$, a variable that is associated with increased smoking for both sexes. Moderate alcohol consumption is thought to reduce the risk of cardiovascular disease and diabetes, especially among individuals with predisposing risk factors, but is thought to increase the risk of cancer and other chronic diseases (26). In some populations, then, moderate consumption could plausibly have a protective net effect on all-cause mortality for men (with their relatively higher burden of cardiovascular and metabolic diseases and related risk factors), yet could have a detrimental net effect for women (27). If this is the case, alcohol consumption could produce confounding bias in different directions for men versus women.

\section{Conclusion}

Reporting these proposed E-values could help characterize the robustness of effect heterogeneity estimates to potential uncontrolled confounding. These results apply to effect 
heterogeneity rather than causal interaction; the Supplement (Section 4) provides E-values for causal interaction that are approximate, "weak" bounds in a sense detailed there. The above E-values for effect heterogeneity could also be applied for causal interaction if one exposure is assumed to be unconfounded (e.g., because it was randomized). 


\section{DATA AVAILABILITY}

All code, materials, and data required to reproduce the applied example are publicly available and documented (https://osf.io/79scv/). Code for the R package EValue and the online tool are open-source (https://github.com/mayamathur/evalue_package and https:// github.com/mayamathur/evalue_website).

\section{ETHICS APPROVAL}

Not applicable.

\section{CONFLiCTS OF INTEREST}

None declared.

\section{Author CONTRibutions}

MBM conceived the research, led theoretical developments, led writing, analyzed the applied example, and wrote code for the R package. LHS contributed to theoretical developments. LHS and all other authors contributed critical intellectual content to writing.

\section{FUNDING}

MBM and TVW were supported by R01 CA222147 and R01 LM013866. MBM was supported by: (1) the NIH-funded Biostatistics, Epidemiology and Research Design (BERD) Shared Resource of Stanford University's Clinical and Translational Education and Research (UL1TR003142); (2) the Biostatistics Shared Resource (BSR) of the NIH-funded Stanford Cancer Institute (P30CA124435); and (3) the Quantitative Sciences Unit through the Stanford Diabetes Research Center (P30DK116074). KY was supported by Brigham and Women's 
Hospital Department of Medicine Fellowship Award and K23AR076453 (NIAMS). The funders had no role in the design, conduct, or reporting of this research.

\section{REFERENCES}

[1] Tyler J VanderWeele. Explanation in causal inference: methods for mediation and interaction. Oxford University Press, 2015.

[2] Tyler J VanderWeele. On the distinction between interaction and effect modification. Epidemiology, 20(6):863-871, 2009.

[3] Tyler J VanderWeele, Bhramar Mukherjee, and Jinbo Chen. Sensitivity analysis for interactions under unmeasured confounding. Statistics in Medicine, 31(22):2552-2564, 2012.

[4] Tyler J VanderWeele and Peng Ding. Sensitivity analysis in observational research: Introducing the E-value. Annals of Internal Medicine, pages 268-274, 2017.

[5] Peng Ding and Tyler J VanderWeele. Sensitivity analysis without assumptions. Epidemiology, 27(3):368, 2016.

[6] Tyler J VanderWeele, Peng Ding, and Maya Mathur. Technical considerations in the use of the E-value. Journal of Causal Inference, 7(2), 2019.

[7] Tyler J VanderWeele and Maya B Mathur. Commentary: developing best-practice guidelines for the reporting of E-values. International Journal of Epidemiology, 49(5):1495$1497,2020$.

[8] Charles Poole. Commentary: Continuing the E-value's post-publication peer review. International Journal of Epidemiology, 49(5):1497-1500, 2020. 
[9] Sander Greenland. Commentary: An argument against E-values for assessing the plausibility that an association could be explained away by residual confounding. International Journal of Epidemiology, 49(5):1501-1503, 2020.

[10] John PA Ioannidis, Yuan Jin Tan, and Manuel R Blum. Limitations and misinterpretations of E-values for sensitivity analyses of observational studies. Annals of Internal Medicine, 170(2):108-111, 2019.

[11] Tyler J VanderWeele. Are Greenland, Ioannidis, and Poole opposed to the Cornfield conditions? A defense of the E-value. Under review.

[12] Tyler J VanderWeele, Maya B Mathur, and Ying Chen. Outcome-wide longitudinal designs for causal inference: a new template for empirical studies. Statistical Science, 35(3):437-466, 2020.

[13] Maya B Mathur and Tyler J VanderWeele. Sensitivity analysis for unmeasured confounding in meta-analyses. Journal of the American Statistical Association, 115(529):163-172, 2020.

[14] Louisa H Smith, Maya B Mathur, and Tyler J VanderWeele. Multiple-bias sensitivity analysis using bounds. Epidemiology, 32(5):625-634, 2021.

[15] Jerome Cornfield, William Haenszel, E Cuyler Hammond, Abraham M Lilienfeld, Michael B Shimkin, and Ernst L Wynder. Smoking and lung cancer: recent evidence and a discussion of some questions. Journal of the National Cancer Institute, 22:173-203, 1959.

[16] James J Schlesselman. Assessing effects of confounding variables. American Journal of Epidemiology, 108(1):3-8, 1978.

[17] Maya B Mathur, Peng Ding, Corinne A Riddell, and Tyler J VanderWeele. Website and R package for computing E-values. Epidemiology, 29(5):e45-e47, 2018. 
[18] Ariel Linden, Maya B Mathur, and Tyler J VanderWeele. Conducting sensitivity analysis for unmeasured confounding in observational studies using E-values: the evalue package. The Stata Journal, 20(1):162-175, 2020.

[19] Tyler J VanderWeele, Timothy L Lash, and Kenneth J Rothman. Analysis of interaction. In Modern epidemiology. Wolters Kluwer, 4 edition, 2021.

[20] L Letenneur, J Launer, K Andersen, ME Dewey, A Ott, JRM Copeland, JF Dartigues, P Kragh-Sorensen, M Baldereschi, C Brayne, et al. Education and risk for Alzheimer's disease: Sex makes a difference. EURODEM pooled analyses. American Journal of Epidemiology, 151(11):1064-1071, 2000.

[21] JE Winter, RJ MacInnis, and CA Nowson. The influence of age on the BMI and all-cause mortality association: A meta-analysis. The Journal of Nutrition, Health $\mathcal{S}^{3}$ Aging, 21(10):1254-1258, 2017.

[22] Maya B Mathur and Tyler J VanderWeele. Methods to address confounding and other biases in meta-analyses: Review and recommendations. Annual Review of Public Health, $43(1)$, in press.

[23] TJ VanderWeele, MB Mathur, and Peng Ding. Correcting misinterpretations of the E-value. Annals of Internal Medicine, 2019.

[24] Maya B Mathur and Tyler J VanderWeele. How to report E-values for meta-analyses: Recommended improvements and additions to the new GRADE approach. 2021. Preprint retrieved from https://osf.io/xb25k.

[25] Xiang Zhang, James D Stamey, and Maya B Mathur. Assessing the impact of unmeasured confounders for credible and reliable real-world evidence. Pharmacoepidemiology and Drug Safety, 29(10):1219-1227, 2020. 
[26] Max G Griswold, Nancy Fullman, Caitlin Hawley, Nicholas Arian, Stephanie RM Zimsen, Hayley D Tymeson, Vidhya Venkateswaran, Austin Douglas Tapp, Mohammad H Forouzanfar, Joseph S Salama, et al. Alcohol use and burden for 195 countries and territories, 1990-2016: a systematic analysis for the global burden of disease study 2016. The Lancet, 392(10152):1015-1035, 2018.

[27] Ian R White, Dan R Altmann, and Kiran Nanchahal. Alcohol consumption and mortality: modelling risks for men and women at different ages. BMJ, 325(7357):191, 2002. 


\title{
Supplement
}

\author{
Contents
}

$\begin{array}{lll}1 & \text { A note on sign assumptions } & 2\end{array}$

$\begin{array}{lll}2 & \text { E-values for multiplicative effect heterogeneity } & 2\end{array}$

2.1 Potentially bidirectional confounding . . . . . . . . . . . . . . 2

2.2 Unidirectional confounding . . . . . . . . . . . . . . . . . 3

2.3 Analogs to Cornfield-Schlesselman conditions . . . . . . . . . . . . . . . . 4

\begin{tabular}{|ccc}
\hline 3 & E-values for additive effect heterogeneity & 6
\end{tabular}

3.1 Potentially bidirectional confounding . . . . . . . . . . . . . 6

3.1 .1 E-value for the confidence intervall . . . . . . . . . . . . . . . . . . . . 7

3.2 Unidirectional confounding . . . . . . . . . . . . . . . . 7

4 A "weak" E-value for multiplicative causal interaction 10 


\section{A NOTE ON SIGN ASSUMPTIONS}

As noted in the main text, we assume throughout all proofs that the estimated effect is larger for stratum $Z=1$ than for $Z=0$ in that $R R_{E H}^{c}>1$ if working on the multiplicative scale or $I C^{c}>0$ if working on the the additive scale. If the opposite holds, one can simply reverse the coding of $Z$ before applying any of the results below.

\section{E-VALUES FOR MULTIPLICATIVE EFFECT HETEROGENEITY}

\subsection{Potentially bidirectional confounding}

Let $U_{1}$ and $U_{0}$ be sets of uncontrolled variables that would suffice to control for confounding of the $X-Y$ relationship within the $Z=1$ and $Z=0$ strata, respectively, such that $Y_{x} \amalg X \mid\left\{U_{1}, Z=1\right\}$ and $Y_{x} \amalg X \mid\left\{U_{0}, Z=0\right\}$. Let the causal $R R$ within stratum $Z=1$ be:1

$$
\begin{aligned}
R R_{X Y \mid Z=1}^{t} & =E\left[Y_{1} \mid Z=1\right] / E\left[Y_{0} \mid Z=1\right] \\
& =\frac{\sum_{u} E\left[Y \mid Z=1, X=1, U_{1}=u\right] P\left(U_{1}=u \mid Z=1\right)}{\sum_{u} E\left[Y \mid Z=1, X=0, U_{1}=u\right] P\left(U_{1}=u \mid Z=1\right)}
\end{aligned}
$$

Its confounded counterpart is:

$$
\begin{aligned}
R R_{X Y \mid Z=1}^{c} & =E[Y \mid Z=1, X=1] / E[Y \mid Z=1, X=0] \\
& =\frac{\sum_{u} E[Y \mid Z=1, X=1, U=u] P(U=u \mid Z=1, X=1)}{\sum_{u} E[Y \mid Z=1, X=0, U=u] P(U=u \mid Z=1, X=0)}
\end{aligned}
$$

and likewise for stratum $Z=0$ except with confounders $U_{0}$ in place of $U_{1}$. Again, any confounders that are measured and controlled can also be conditioned throughout, but we suppress this notation for brevity.

Within stratum $z$, define the bias factor $B_{z}=R R_{X Y \mid Z=z}^{c} / R R_{X Y \mid Z=z}^{t} \in(0, \infty)$, and define the sensitivity parameters $a_{z}=R R_{X U \mid Z=z}$ and $b_{z}=R R_{U Y \mid Z=z}$, as given in the main text. For the general case in which the direction of confounding in each stratum is considered unknown, a simple application of Ding \& VanderWeele (2016a)'s bounding factor within each stratum yields the bounds:

$$
B F^{-1}\left(a_{z}, b_{z}\right) \leq B_{z} \leq B F\left(a_{z}, b_{z}\right)
$$

where $B F\left(a_{z}, b_{z}\right)=\left(a_{z} b_{z}\right) /\left(a_{z}+b_{z}-1\right) \geq 1$.

\footnotetext{
${ }^{1}$ For full generality, the summations could be replaced with integrals to accommodate continuous confounders.
} 
As follows, the multiplicative effect heterogeneity risk ratio, $R R_{E H}^{t}$, is minimized when $R R_{X Y \mid Z=1}^{c}$ is positively biased and $R R_{X Y \mid Z=0}^{c}$ is negatively biased:

$$
\begin{aligned}
R R_{E H}^{t} & =R R_{X Y \mid Z=1}^{t} / R R_{X Y \mid Z=0}^{t} \\
& =\frac{R R_{X Y \mid Z=1}^{c} / B_{1}}{R R_{X Y \mid Z=0}^{c} / B_{0}} \\
& \geq \frac{R R_{X Y \mid Z=1}^{c} / B F\left(a_{1}, b_{1}\right)}{R R_{X Y \mid Z=0}^{c} \times B F\left(a_{0}, b_{0}\right)} \\
& =R R_{E H}^{c} / B F_{E H}
\end{aligned}
$$

where

$$
\begin{aligned}
B F_{E H} & =B F\left(a_{0}, b_{0}\right) \times B F\left(a_{1}, b_{1}\right) \\
& =\frac{a_{1} b_{1} a_{0} b_{0}}{\left(a_{1}+b_{1}-1\right)\left(a_{0}+b_{0}-1\right)}
\end{aligned}
$$

To obtain the E-value required to reduce the effect heterogeneity point estimate to $R R_{E H}^{t}$, let $m=\max \left\{a_{1}, b_{1}, a_{0}, b_{0}\right\}$. Because $B F_{E H}$ is increasing in $m$, we have:

$$
\begin{aligned}
B F_{E H} & \leq \frac{m^{4}}{(2 m-1)^{2}} \\
m & \geq \sqrt{\frac{R R_{E H}^{c}}{R R_{E H}^{t}}}+\sqrt{\frac{R R_{E H}^{c}}{R R_{E H}^{t}}-\sqrt{\frac{R R_{E H}^{c}}{R R_{E H}^{t}}}}
\end{aligned}
$$

where the latter bound is the E-value. Setting $R R_{E H}^{t}=1$ yields the result in the main text regarding shifting the estimate to the null.

\subsection{Unidirectional confounding}

The above development of $B F_{E H}$ involved applying the bound in Eq. 2.1) independently to each stratum, such that the bound on $B F_{E H}$ arose under confounding whose direction differed between strata of $Z$. Suppose we more stringently assume that confounding operates in the same direction in each stratum ("unidirectional confounding"). Because the bounds on the multiplicative scale are symmetric for the 2 strata, we consider positive bias in each stratum without loss of generality, such that:

$$
1 \leq B_{z} \leq B F\left(a_{z}, b_{z}\right)
$$


producing the bound

$$
\begin{aligned}
R R_{E H}^{t} & \geq \frac{R R_{X Y \mid Z=1}^{c} \times B F\left(a_{1}, b_{1}\right)}{R R_{X Y \mid Z=0}^{c} \times B F\left(a_{0}, b_{0}\right)} \\
& =R R_{E H}^{c} / B F_{E H}^{\prime}
\end{aligned}
$$

where

$$
\begin{aligned}
B F_{E H}^{\prime} & =B F\left(a_{1}, b_{1}\right) / B F\left(a_{0}, b_{0}\right) \\
& =\frac{a_{1} b_{1}}{a_{1}+b_{1}-1} \div \frac{a_{0} b_{0}}{a_{0}+b_{0}-1}
\end{aligned}
$$

Since $B F_{E H}$ is increasing in $a_{1}$ and $b_{1}$ and decreasing in $a_{0}$ and $b_{0}$, the E-value arises when the $Z=0$ stratum is unbiased, such that $a_{0}=b_{0}=1$ :

$$
\begin{aligned}
B F_{E H}^{\prime} & \leq \frac{m^{2}}{(2 m-1)^{2}} \\
m & \geq \frac{R R_{E H}^{c}}{R R_{E H}^{t}}+\sqrt{\frac{R R_{E H}^{c}}{R R_{E H}^{t}}\left(\frac{R R_{E H}^{c}}{R R_{E H}^{t}}-1\right)}
\end{aligned}
$$

Eq. (2.3) represents the E-value under unidirectional confounding; the expression in the main text again arises from setting $R R_{E H}^{t}=1$. Again, this result holds without loss of generality to the symmetric case in which, instead, the $Z=0$ stratum is negatively biased the $Z=1$ stratum is unbiased.

\subsection{Analogs to Cornfield-Schlesselman conditions}

Here we establish connections between our results and classical sensitivity analyses for uncontrolled confounding. By assuming the presence of a single, binary uncontrolled confounder, $U$, Cornfield et al. (1959) developed well-known sensitivity analyses for uncontrolled confounding of a total effect on the $R R$ scale, here termed $R R_{X Y}^{c}$. In a generalization and extension of those results, Schlesselman (1978) further assumed that $U$ does not interact with $X$, and he defined the following sensitivity parameters:

$$
\begin{aligned}
& R R_{X U}=E[U \mid X=1] / E[U \mid X=0] \\
& R R_{U Y}=E[Y \mid X=0, U=1] / E[U \mid X=0, U=0]
\end{aligned}
$$

(These sensitivity parameters are special cases of those defined in Ding \& VanderWeele (2016b) and in the present main text.) Under these assumptions regarding $U$, Cornfield et al. (1959) and Schlesselman (1978) respectively showed that if $U$ fully explains away $R R_{X Y}^{c}$, then each sensitivity parameter can be separately bounded by $R R_{X U} \geq R R_{X Y}^{c}$ and $R R_{U Y} \geq R R_{X Y}^{c}$. Ding \& VanderWeele (2016a) showed that first of these classical bounds 
arises as a special case of Ding \& VanderWeele (2016a)'s own bound, $B F(a, b)$, when no restrictions are placed on $R R_{U Y}$ (i.e., when letting $R R_{U Y} \rightarrow \infty$ ). Similarly, the second classical bound, $R R_{U Y} \geq R R_{X Y}^{c}$, holds as $R R_{X U} \rightarrow \infty$ (Ding \& VanderWeele, 2016a).

These Cornfield-Schlesselman conditions for total effects essentially provide a lower bound on a given sensitivity parameter $\left(R R_{X U}\right.$ or $\left.R R_{U Y}\right)$ when placing no restrictions on the other sensitivity parameter. For effect heterogeneity, one might define analogs to CornfieldSchlesselman conditions as lower bounds on each of $R R_{X U \mid Z=1}, R R_{X U \mid Z=0}, R R_{U Y \mid Z=1}$, and $R R_{U Y \mid Z=0}$, each time placing no restrictions on the other 3 parameters. However, because it is possible for all of the bias in $R R_{E H}^{c}$ to arise from bias in only one stratum of $Z$, each of these bounds is simply 1, which is not informative. For example, the lower bound on $R R_{X U \mid Z=1}$ would arise when there is no bias in stratum $Z=1$, and instead all of the bias in $R R_{E H}^{c}$ arises from bias in the stratum $Z=0$.

Instead, we suggest defining the following analogs to Cornfield-Schlesselman conditions for the setting of multiplicative effect heterogeneity. First, we provide a lower bound on $\max _{z} R R_{X U \mid Z=z}$, while placing no restrictions on $R R_{U Y \mid Z=0}$ or $R R_{U Y \mid Z=1}$. This bound answers the question, "How large must $R R_{X U \mid Z=z}$ be in at least one stratum of $Z$ in order to fully explain away $R R_{E H}^{c}$ ?" Second, we provide a lower bound on $\max _{z} R R_{U Y \mid Z=z}$, while placing no restrictions on $R R_{X U \mid Z=0}$ or $R R_{X U \mid Z=1}$. This bound answers the question, "How large must $R R_{U Y \mid Z=z}$ be in at least one stratum of $Z$ in order to fully explain away $R R_{E H}^{c}$ ?"

The bound on $\max _{z} R R_{X U \mid Z=z}$ can be obtained by letting $b_{0} \rightarrow \infty$ and $b_{1} \rightarrow \infty$ in Eq. (2.2):

$$
\begin{aligned}
\lim _{b_{0}, b_{1} \rightarrow \infty} B F_{E H} & =\lim _{b_{0}, b_{1} \rightarrow \infty}\left\{\frac{a_{1} b_{1}}{a_{1}+b_{1}-1} \cdot \frac{a_{0} b_{0}}{a_{0}+b_{0}-1}\right\} \\
& =\lim _{b_{0}, b_{1} \rightarrow \infty}\left\{\frac{a_{1}}{a_{1} / b_{1}+1-1 / b_{1}} \cdot \frac{a_{0}}{a_{0} / b_{0}+1-1 / b_{0}}\right\} \\
& =a_{1} a_{0} \\
& \leq\left(\max \left\{a_{1}, a_{0}\right\}\right)^{2}
\end{aligned}
$$

Symmetrically, the bound on $\max _{z} R R_{X Y \mid Z=z}$ can be obtained by instead letting $a_{0} \rightarrow \infty$ and $a_{1} \rightarrow \infty$. The analogs to the Cornfield conditions are therefore:

$$
\begin{aligned}
& \max _{z} R R_{X U \mid Z=z} \geq \sqrt{R R_{E H}^{c}} \\
& \max _{z} R R_{U Y \mid Z=z} \geq \sqrt{R R_{E H}^{c}}
\end{aligned}
$$

In contrast to the original Cornfield-Schlesselman conditions, these results do not require the assumption that $U$ is a single, binary unmeasured confounder, nor the assumption that $U$ does not interact with $X$. 


\section{E-VALUES FOR ADDITIVE EFFECT HETEROGENEITY}

\subsection{Potentially bidirectional confounding}

Define the causal risk difference in stratum $Z=z$ and its confounded counterpart as:

$$
\begin{aligned}
& R D_{X Y \mid Z=z}^{t}=E\left[Y_{1} \mid Z=z\right]-E\left[Y_{0} \mid Z=z\right] \\
& R D_{X Y \mid Z=z}^{c}=E[Y \mid Z=z, X=1]-E[Y \mid Z=z, X=0]
\end{aligned}
$$

We take the causal estimand to be the additive interaction contrast (VanderWeele et al., 2021):

$$
I C^{t}=R D_{X Y \mid Z=1}^{t}-R D_{X Y \mid Z=0}^{t}
$$

Ding \& VanderWeele (2016a) showed that each within-stratum risk difference can be bounded below (for positive bias) or above (for negative bias) by:

$$
\begin{aligned}
& R D_{X Y \mid Z=z}^{t} \geq\left\{p_{z 1}-p_{z 0} \times B F\left(a_{z}, b_{z}\right)\right\}\left\{f_{z}+\frac{1-f_{z}}{B F\left(a_{z}, b_{z}\right)}\right\} \\
& R D_{X Y \mid Z=z}^{t} \leq\left\{p_{z 1} \times B F\left(a_{z}, b_{z}\right)-p_{z 0}\right\}\left\{f_{z}+\frac{1-f_{z}}{B F\left(a_{z}, b_{z}\right)}\right\}
\end{aligned}
$$

where $f_{z}=E[X \mid Z=z]$ and $p_{z x}=E[Y \mid Z=z, X=x]$. The lower bound in Eq. (3.1) is decreasing in $B F\left(a_{z}, b_{z}\right)$, and the upper bound in Eq. (3.2) is increasing in $B F\left(a_{z}, b_{z}\right)$, which can be verified by taking first derivatives. Thus, under potentially bidirectional confounding, the lower bound on $I C^{t}$ arises when $R D_{X Y \mid Z=1}^{c}$ is positively biased and $R D_{X Y \mid Z=0}^{c}$ is negatively biased:

$$
\begin{aligned}
I C^{t} & \geq\left\{p_{11}-p_{10} \times B F\left(a_{1}, b_{1}\right)\right\}\left\{f_{1}+\frac{1-f_{1}}{B F\left(a_{1}, b_{1}\right)}\right\}-\left\{p_{01} \times B F\left(a_{0}, b_{0}\right)-p_{00}\right\}\left\{f_{0}+\frac{1-f_{0}}{B F\left(a_{0}, b_{0}\right)}\right\} \\
& \geq\left\{p_{11}-p_{10} \times \frac{m^{2}}{(2 m-1)^{2}}\right\}\left\{f_{1}+\frac{1-f_{1}}{\frac{m^{2}}{(2 m-1)^{2}}}\right\}-\left\{p_{01} \times \frac{m^{2}}{(2 m-1)^{2}}-p_{00}\right\}\left\{f_{0}+\frac{1-f_{0}}{\frac{m^{2}}{(2 m-1)^{2}}}\right\}
\end{aligned}
$$

where, again, $m=\max \left\{a_{1}, b_{1}, a_{0}, b_{0}\right\}$. Solving for $m$ yields the E-value required to reduce the causal effect to $I C^{t}$ :

$$
\begin{aligned}
m \geq g\left(\frac{1}{2\left(f_{1} p_{10}+f_{0} p_{01}\right)}\right. & \left(\sqrt{\left\{I C^{t}+\gamma\right\}^{2}+4\left(p_{10} f_{1}+p_{01} f_{0}\right)\left(p_{11}\left[1-f_{1}\right]+p_{00}\left[1-f_{0}\right]\right)}\right. \\
& \left.\left.-\left\{I C^{t}+\gamma\right\}\right)\right)
\end{aligned}
$$

where $g(w)=w+\sqrt{w(w-1)}$ and $\gamma=p_{10}\left(1-f_{1}\right)-p_{11} f_{1}+p_{01}\left(1-f_{0}\right)-p_{00} f_{0}$. 


\subsubsection{E-value for the confidence interval}

When the confounded sample estimate $\widehat{R D}_{X Y \mid Z=z}^{c}$ is positively biased, the variance of the bias-corrected sample estimate, $\widehat{\operatorname{Var}}\left(\widehat{R D}_{X Y \mid Z=z}^{t}\right)$, can be approximated via the delta method (Ding \& VanderWeele, 2016a):

$$
\begin{aligned}
\widehat{\operatorname{Var}}\left(\widehat{R D}_{X Y \mid Z=z}^{t}\right) & =\left(\widehat{v}_{z 1}+\widehat{v}_{z 0} \times B F\left(a_{z}, b_{z}\right)^{2}\right)\left(\widehat{f}_{z}+\frac{1-\widehat{f}_{z}}{B F\left(a_{z}, b_{z}\right)}\right)^{2} \\
& +\left(\widehat{p}_{z 1}-\widehat{p}_{z 0} \times B F\left(a_{z}, b_{z}\right)\right)^{2}\left(1-\frac{1}{B F\left(a_{z}, b_{z}\right)}\right)^{2} \widehat{v}_{z}
\end{aligned}
$$

where $\widehat{v}_{z}=\widehat{\operatorname{Var}}\left(\widehat{f}_{z}\right)$ and $\widehat{v}_{z x}=\widehat{\operatorname{Var}}\left(\widehat{p}_{z x}\right)$. When $\widehat{R D}_{X Y \mid Z=z}^{t}$ is instead negatively biased, then by symmetry, one can essentially recode the exposure by reversing the roles of $\widehat{p}_{z 0}$ and $\widehat{p}_{z 1}$ and reversing the roles of $\widehat{v}_{z 0}$ and $\widehat{v}_{z 1}$ in the above expression. Thus, the variance of $\operatorname{Var}\left(\widehat{I C}^{t}\right)$ can be approximated as:

$$
\begin{aligned}
\widehat{\operatorname{Var}}\left(\widehat{I C}^{t}\right) & =\widehat{\operatorname{Var}}\left(\widehat{R D}_{X Y \mid Z=1}^{t}\right)+\widehat{\operatorname{Var}}\left(\widehat{R D}_{X Y \mid Z=0}^{t}\right) \\
& =\left(\widehat{v}_{11}+\widehat{v}_{10} \times B F\left(a_{1}, b_{1}\right)^{2}\right)\left(\widehat{f}_{1}+\frac{1-\widehat{f}_{1}}{B F\left(a_{1}, b_{1}\right)}\right)^{2}+\left(\widehat{p}_{11}-\widehat{p}_{10} \times B F\left(a_{1}, b_{1}\right)\right)^{2}\left(1-\frac{1}{B F\left(a_{1}, b_{1}\right)}\right)^{2} \widehat{v}_{1} \\
& +\left(\widehat{v}_{01} \times B F\left(a_{0}, b_{0}\right)^{2}+\widehat{v}_{00}\right)\left(\widehat{f}_{0}+\frac{1-\widehat{f}_{0}}{B F\left(a_{0}, b_{0}\right)}\right)^{2}+\left(\widehat{p}_{00}-\widehat{p}_{01} \times B F\left(a_{0}, b_{0}\right)\right)^{2}\left(1-\frac{1}{B F\left(a_{0}, b_{0}\right)}\right)^{2} \widehat{v}_{0}
\end{aligned}
$$

To obtain the E-value required to shift the lower confidence interval limit to $I C_{l b}^{t}$ (for example, $I C_{l b}^{t}=0$ for the null), one could set $B F\left(a_{1}, b_{1}\right)=B F\left(a_{0}, b_{0}\right)=\frac{m^{2}}{2 m-1}$ in Eq. 3.4 and numerically solve the following expression for $m$ :

$$
\widehat{I C}_{l b}^{t}=\widehat{I C}^{t}-\Phi(1-\alpha / 2) \sqrt{\widehat{\operatorname{Var}}\left(\widehat{I C}^{t}\right)}
$$

where $\Phi$ is the standard normal cumulative distribution function, $1-\alpha$ is the level of the confidence interval, and $\widehat{I C}^{t}$ is obtained from substituting sample prevalence estimates into Eq. (3.3).

\subsection{Unidirectional confounding}

If the confounding bias is assumed to be unidirectional, there are 3 cases: both strata are positively biased, both strata are negatively biased, or both strata are biased in the same unknown direction. For additive effect heterogeneity, unlike for multiplicative effect 
heterogeneity, these cases are no longer symmetric because the bounds on each stratum's risk difference depend on stratum-specific nuisance parameters.

\section{Case 1: Both strata are positively biased}

If both strata are positively biased, $I C^{t}$ is bounded by:

$$
\begin{aligned}
I C^{t} & \geq\left\{p_{11}-p_{10} \times B F\left(a_{1}, b_{1}\right)\right\}\left\{f_{1}+\frac{1-f_{1}}{B F\left(a_{1}, b_{1}\right)}\right\} \\
& -\left\{p_{01}-p_{00} \times B F\left(a_{0}, b_{0}\right)\right\}\left\{f_{0}+\frac{1-f_{0}}{B F\left(a_{0}, b_{0}\right)}\right\}
\end{aligned}
$$

The bias of $I C^{c}$ is most severe when the right-hand side is minimized. To that end, the right-hand side is decreasing $B F\left(a_{1}, b_{1}\right)$ and increasing in $B F\left(a_{0}, b_{0}\right)$, so is minimized when $B F\left(a_{0}, b_{0}\right)=1$ (i.e., when the $Z=0$ stratum is unbiased). Therefore, for this case, the E-value required to reduce the causal effect to $I C^{t}$ is simply equivalent to the E-value required to decrease $R D_{X Y \mid Z=1}^{t}$ to $I C^{t}+R D_{X Y \mid Z=0}^{t}$, with the bound attained when $\widehat{R D}_{X Y \mid Z=0}^{c}$ is unbiased:

$$
\begin{aligned}
I C^{t} \geq \quad & \left\{p_{11}-p_{10} \times B F\left(a_{1}, b_{1}\right)\right\}\left\{f_{1}+\frac{1-f_{1}}{B F\left(a_{1}, b_{1}\right)}\right\}-\left(p_{01}-p_{00}\right) \\
I C^{t}+\left(p_{01}-p_{00}\right) \geq \quad & \left\{p_{11}-p_{10} \times B F\left(a_{1}, b_{1}\right)\right\}\left\{f_{1}+\frac{1-f_{1}}{B F\left(a_{1}, b_{1}\right)}\right\} \\
m_{1} \geq g\left(\frac{1}{2 f_{1} p_{10}}\right. & \left(\sqrt{\left\{I C^{t}+\left(p_{01}-p_{00}\right)+\lambda_{1}\right\}^{2}+4 p_{11} p_{10} f_{1}\left(1-f_{1}\right)}\right. \\
& \left.\left.-\left\{I C^{t}+\left(p_{01}-p_{00}\right)+\lambda_{1}\right\}\right)\right)
\end{aligned}
$$

where $m_{1}=\max \left\{a_{1}, b_{1}\right\}, \lambda_{1}=p_{10}\left(1-f_{1}\right)-p_{11} f_{1}$ and, again, $g(w)=w+\sqrt{w(w-1)}$.

From Eq. 3.6), the variance of $\widehat{I C}^{t}$ in the bounding case (when only the $Z=1$ stratum is biased) can be approximated as (Ding \& VanderWeele, 2016a):

$$
\begin{aligned}
\widehat{\operatorname{Var}}\left(\widehat{I C}^{t}\right) & =\left(\widehat{v}_{11}+\widehat{v}_{10} \times\left(\frac{m^{2}}{(2 m-1)^{2}}\right)^{2}\right)\left(\widehat{f}_{1}+\frac{1-\widehat{f}_{1}}{\frac{m^{2}}{(2 m-1)^{2}}}\right)^{2} \\
& +\left(\widehat{p}_{11}-\widehat{p}_{10} \times \frac{m^{2}}{(2 m-1)^{2}}\right)^{2}\left(1-\frac{1}{\frac{m^{2}}{(2 m-1)^{2}}}\right)^{2} \widehat{v}_{1} \\
& +\widehat{v}_{01}+\widehat{v}_{00}
\end{aligned}
$$

To obtain the E-value required to shift the lower confidence interval limit to $I C_{l b}^{t}$, one 
could again numerically solve Eq. 3.5 for $m$, now with $\widehat{I C}^{t}$ given by Eq. 3.6 with $B F\left(a_{1}, b_{1}\right)=\frac{m^{2}}{(2 m-1)^{2}}$ and with $\widehat{\operatorname{Var}}\left(\widehat{I C}^{t}\right)$ given by Eq. 3.8.

\section{Case 2: Both strata are negatively biased}

If both strata are negatively biased, $I C^{t}$ is bounded by:

$$
\begin{aligned}
I C^{t} & \geq\left\{p_{11} \times B F\left(a_{1}, b_{1}\right)-p_{10}\right\}\left\{f_{1}+\frac{1-f_{1}}{B F\left(a_{1}, b_{1}\right)}\right\} \\
& -\left\{p_{01} \times B F\left(a_{0}, b_{0}\right)-p_{00}\right\}\left\{f_{0}+\frac{1-f_{0}}{B F\left(a_{0}, b_{0}\right)}\right\}
\end{aligned}
$$

Again, the bias of $I C^{c}$ is most severe when the right-hand side is minimized. The righthand side is increasing in $B F\left(a_{0}, b_{0}\right)$ and decreasing in $B F\left(a_{1}, b_{1}\right)$, so is minimized when $B F\left(a_{1}, b_{1}\right)=1$ (i.e., the $Z=1$ stratum is unbiased). Therefore, for this case, the E-value required to reduce the causal effect to $I C^{t}$ is simply equivalent to the E-value required to increase $R D_{X Y \mid Z=0}^{t}$ to $R D_{X Y \mid Z=1}^{t}-I C^{t}$, with the bound attained when $\widehat{R D}_{X Y \mid Z=1}^{c}$ is unbiased (Ding \& VanderWeele, 2016a): 2

$$
\begin{aligned}
\left(p_{11}-p_{10}\right)-I C^{t} \leq \quad & \left\{p_{01} \times B F\left(a_{0}, b_{0}\right)-p_{00}\right\}\left\{f_{0}+\frac{1-f_{0}}{B F\left(a_{0}, b_{0}\right)}\right\} \\
m_{0} \geq \frac{1}{2 f_{0} p_{01}} & \left(\sqrt{\left\{\left(I C^{t}-\left(p_{11}-p_{10}\right)\right)+\lambda_{0}\right\}^{2}+4 p_{01} p_{00} f_{0}\left(1-f_{0}\right)}\right. \\
& \left.-\left\{I C^{t}-\left(p_{11}-p_{10}\right)+\lambda_{0}\right\}\right)
\end{aligned}
$$

where $\lambda_{0}=p_{01}\left(1-f_{0}\right)-p_{00} f_{0}$. We use $m_{0}$ here to denote that this E-value applies to the case in which the only the $Z=0$ stratum is biased.

From Eq. 3.9, the variance of $\widehat{I C}^{t}$ in the bounding case in the bounding case (when only the $Z=0$ stratum is biased) can be approximated as (Ding \& VanderWeele, 2016a):

$$
\begin{aligned}
\widehat{\operatorname{Var}}\left(\widehat{I C}^{t}\right) & =\left(\widehat{v}_{01} \times\left(\frac{m^{2}}{(2 m-1)^{2}}\right)^{2}+\widehat{v}_{00}\right)+\left(\widehat{f}_{0}+\frac{1-\widehat{f}_{0}}{\frac{m^{2}}{(2 m-1)^{2}}}\right)^{2} \\
& +\left(\widehat{p}_{00}-\widehat{p}_{01} \times \frac{m^{2}}{(2 m-1)^{2}}\right)^{2}\left(1-\frac{1}{\frac{m^{2}}{(2 m-1)^{2}}}\right)^{2} \widehat{v}_{0} \\
& +\widehat{v}_{11}+\widehat{v}_{10}
\end{aligned}
$$

\footnotetext{
${ }^{2}$ This expression is simply Ding \& VanderWeele (2016a)'s lower bound for the risk difference when the bias is positive except with the roles of $p_{11}$ and $p_{01}$ reversed. This holds because reversing their roles also reverses the sign of risk difference, after which the same bound applies by symmetry.
} 
An E-value for the confidence interval limit could then be obtained numerically, proceeding analogously to the previous case.

\section{Case 3: Both strata are biased in the same unknown direction}

If we assume that confounding is unidirectional but do not assume in which direction the bias operates, then the E-value for the point estimate is the minimum of the E-values given for the 2 cases above in Eq. (3.7) and Eq. (3.10):

$$
m \geq \min \left\{m_{1}, m_{0}\right\}
$$

This E-value arises when either the $Z=1$ stratum is positively biased or the $Z=0$ stratum is negatively biased, and the other stratum is unbiased. When the bound is attained, the stratum that is biased is the one for which, based on its nuisance prevalence parameters $p_{z x}$ and $f_{z}$, a smaller bias factor suffices to produce the required bias in that stratum.

An E-value for the confidence interval limit could similarly be obtained by taking the minimum of the E-values for the confidence interval under Cases 1 and 2 above.

\section{A "WEAK" E-VALUE FOR MULTiPliCATIVE CAUSAL INTERACTION}

Although it is possible to derive a sharp bound for causal interaction, the sharp bound depends on a large number of sensitivity parameters and thus is difficult to apply in practice. An alternative approach is to derive a weaker bound by treating each of the 4 possible joint interventions on $(Z, X)$ as mathematically independent, ignoring logical constraints on the relationships among each joint intervention's sensitivity parameters. We refer to the resulting E-value as "weak", meaning that it results from a non-sharp bound. That is, unlike for the standard E-value, there may be no structure of uncontrolled confounding that is capable of attaining the bound that results from sensitivity parameters corresponding to the weak E-value. However, conversely, if the weak E-value is large given the scientific context and the quality of existing confounding control (VanderWeele \& Mathur, 2020), one could conclude that the causal interaction estimate is fairly robust.

As in the results for effect heterogeneity, we assume that other than the omission of uncontrolled confounder(s), the estimator used to obtain the confounded estimate of causal interaction is correctly specified. For example, analyses of causal interaction typically assume that $X$ does not affect $Z$, nor vice versa. Again, this assumption is required for standard estimates of causal interaction (e.g., statistical interaction coefficients obtained by regression) even if there were no uncontrolled confounding. If instead there is causation between $X$ and $Z$, valid estimators of causal interaction exist, but require decomposing interactions from causal effects arising from mediation between the exposures (VanderWeele, 2014). 
Let $Y_{z x}$ be the potential outcome under the joint intervention $(Z=z, X=x)$ and $R R_{z x}^{t}=$ $E\left[Y_{z x}\right] / E\left[Y_{00}\right]$. For the sensitivity parameters, define

$$
R R_{(Z X) U}=\max _{u, z, x}\left\{\frac{P(U=u \mid Z=z, X=x)}{P(U=u \mid Z=0, X=0)}\right\}
$$

This represents the maximum $R R$ relating a joint intervention to $U$, maximized not only across all possible contrasts in $U$ but also across all possible joint interventions compared to the reference intervention $(Z=0, X=0)$. Similarly, define $R R_{U Y}$ as:

$$
R R_{U Y}=\max _{z, x}\left\{\frac{\max _{u} E[Y \mid U=u, X=x, Z=z]}{\min _{u} E[Y \mid U=u, X=x, Z=z]}\right\}
$$

This represents the maximum $R R$ relating $U$ to $Y$ across all possible joint interventions. Let $\widetilde{B F}=B F\left(R R_{(Z X) U}, R R_{U Y}\right)$, where the tilde denotes a bound that is not sharp, and let $B_{x z}=R R_{x z}^{c} / R R_{x z}^{t}$. We thus have bound:

$$
1 / \widetilde{B F} \leq B_{x z} \leq \widetilde{B F}
$$

Let $m=\max \left\{R R_{(Z X) U}, R R_{U Y}\right\}$ and consider the causal interaction:

$$
\begin{aligned}
R R_{\text {int }}^{t} & =R R_{11}^{t} /\left(R R_{10}^{t} R R_{01}^{t}\right) \\
& \geq \widetilde{B F} /\left(\widetilde{B F}^{-2}\right) \\
& \geq\left(\frac{m^{2}}{2 m-1}\right)^{3} \\
m & \geq \sqrt[3]{\widetilde{B F}}+\sqrt{\sqrt[3]{\widetilde{B F}}}(\sqrt[3]{\widetilde{B F}}-1)
\end{aligned}
$$

The final line is the "weak" E-value. It can be obtained using existing software that calculates E-values for main effects (Linden et al., 2020, Mathur et al., 2018) by simply performing the calculation using $\sqrt[3]{R R_{\text {int }}^{t} \text { rather than } R R_{\text {int }}^{t}}$ itself.

To illustrate the interpretation of this weak E-value, suppose that $R R_{i n t}^{c}=2$, such that the weak E-value is 1.83. Therefore, to explain away the interaction, at least one of the following sensitivity parameters must be at least equal to 1.83: (i) the maximum $R R$ relating any of the 3 possible joint interventions to $U$ and (ii) the maximum $R R$ relating $U$ to $Y$ across the 3 joint intervention strata. Confounding weaker than this could not explain away the interaction, but it is not necessarily the case that there exists a hypothetical structure of uncontrolled confounder(s) of this severity that in fact could explain away the interaction. 


\section{REFERENCES}

Cornfield, J., Haenszel, W., Hammond, E. C., Lilienfeld, A. M., Shimkin, M. B., \& Wynder, E. L. (1959). Smoking and lung cancer: recent evidence and a discussion of some questions. Journal of the National Cancer Institute, 22, 173-203.

Ding, P., \& VanderWeele, T. J. (2016a). Sensitivity analysis without assumptions. Epidemiology, $27(3), 368$.

Ding, P., \& VanderWeele, T. J. (2016b). Sensitivity analysis without assumptions. Epidemiology, $27(3), 368$.

Linden, A., Mathur, M. B., \& VanderWeele, T. J. (2020). Conducting sensitivity analysis for unmeasured confounding in observational studies using E-values: the evalue package. The Stata Journal, 20(1), 162-175.

Mathur, M. B., Ding, P., Riddell, C. A., \& VanderWeele, T. J. (2018). Website and R package for computing E-values. Epidemiology, 29(5), e45-e47.

Schlesselman, J. J. (1978). Assessing effects of confounding variables. American Journal of Epidemiology, 108(1), 3-8.

VanderWeele, T. J. (2014). A unification of mediation and interaction: a four-way decomposition. Epidemiology, 25(5), 749.

VanderWeele, T. J., Lash, T. L., \& Rothman, K. J. (2021). Analysis of interaction. In Modern epidemiology (4th ed.). Wolters Kluwer.

VanderWeele, T. J., \& Mathur, M. B. (2020). Commentary: developing best-practice guidelines for the reporting of E-values. International Journal of Epidemiology, 49(5), 1495-1497. 